\title{
Asymptotic behavior of solutions to a class of semilinear parabolic equations
}

\author{
Wei Guo ${ }^{1}$, Xinyue Wang ${ }^{2}$ and Mingjun Zhou ${ }^{3 *}$ (I)
}

"Correspondence:

zhoumj1989@hotmail.com

${ }^{3}$ School of Mathematics, Jilin

University, Changchun 130012,

China

Full list of author information is

available at the end of the article

\begin{abstract}
This paper concerns the asymptotic behavior of solutions to the homogeneous Neumann exterior problems of a class of semilinear parabolic equations with convection and reaction terms. The critical Fujita exponents theorems are established. It is shown that the global existence and blow-up of solutions depends on the reaction term, the convection term and the spatial dimension.
\end{abstract}

MSC: $35 \mathrm{~K} 65 ; 35 \mathrm{~B} 33$

Keywords: convection; reaction; asymptotic behavior

\section{Introduction}

In this paper, we consider the asymptotic behavior of solutions to the following problem:

$$
\begin{aligned}
& \frac{\partial u}{\partial t}=\Delta u+\lambda_{1} \frac{x}{|x|^{2}} \cdot \nabla u+|x|^{\lambda_{2}} t^{\lambda_{3}} u^{p}, \quad x \in \mathbb{R}^{n} \backslash \bar{B}_{1}, t>0, \\
& \frac{\partial u}{\partial v}=0, \quad x \in \partial B_{1}, t>0 \\
& u(x, 0)=u_{0}(x), \quad x \in \mathbb{R}^{n} \backslash \bar{B}_{1},
\end{aligned}
$$

where $\lambda_{1}, \lambda_{2}, \lambda_{3} \geq 0, p>1,0 \leq u_{0} \in C\left(\mathbb{R}^{n} \backslash B_{1}\right) \cap L^{\infty}\left(\mathbb{R}^{n} \backslash B_{1}\right), B_{1}$ is the unit ball in $\mathbb{R}^{n}$.

The studies on asymptotic behavior of solutions to diffusion equations with nonlinear reaction was begun in 1966 by Fujita in [1], where it was proved that for the Cauchy problem to the semilinear equation

$$
\frac{\partial u}{\partial t}=\Delta u+u^{p}, \quad x \in \mathbb{R}^{n}, t>0
$$

the problem does not have any nontrivial, nonnegative global solution if $1<p<p_{c}=1+2 / n$, whereas if $p>p_{c}$, there exist both global (with small data) and non-global (with large initial data) solutions. This result shows that the exponent $p$ of the nonlinear reaction affects the properties of solutions directly. We call $p_{c}$ the critical Fujita exponent and such a result a blow-up theorem of Fujita type.

The elegant work of Fujita revealed a new phenomenon of nonlinear evolution equations. There have been a number of extensions of Fujita's results in several directions since

(c) 2016 Guo et al. This article is distributed under the terms of the Creative Commons Attribution 4.0 International License (http://creativecommons.org/licenses/by/4.0/), which permits unrestricted use, distribution, and reproduction in any medium, provided you give appropriate credit to the original author(s) and the source, provide a link to the Creative Commons license, and indicate if changes were made. 
then, including similar results for numerous of quasilinear parabolic equations and systems in various of geometries (whole spaces, cones, and exterior domains) with nonlinear reactions or nonhomogeneous boundary conditions, and even degenerate equations in domains with non-compact boundary [2,3]. We refer to the survey papers $[4,5]$ and the references therein, and more recent work [6-20]. Qi [8] considered the Cauchy problem of (1) without convection, i.e. $\lambda_{1}=0$, and proved that the Fujita exponent is

$$
p_{c}=1+\frac{2+\lambda_{2}+2 \lambda_{3}}{n} \text {. }
$$

Zheng and Wang [15] studied the homogeneous Neumann exterior problem (1)-(3) with the special case $\lambda_{3}=0$, and proved that the Fujita exponent is

$$
p_{c}=1+\frac{2+\lambda_{2}}{n+\lambda_{1}}
$$

In this paper, we consider the homogeneous Neumann exterior problem (1)-(3), and prove that the Fujita exponent is

$$
p_{c}=1+\frac{2+\lambda_{2}+2 \lambda_{3}}{n+\lambda_{1}}
$$

which depends on $n, \lambda_{1}, \lambda_{2}, \lambda_{3}$. The technique used in this paper for establishing the Fujita type results for the problem (1)-(3) is mainly inspired by $[8,10,11]$. To prove the blow-up of solutions, we will determine the interactions among the diffusion terms, convection terms and reaction terms by a series of precise integral estimates instead of pointwise comparisons. Here, we also need to construct some subsolutions to get some integral estimates of the solutions. As to the existence of global solutions, we construct some global self-similar supersolutions.

This paper is arranged as follows. The main results of the paper are stated in Section 2 and their proofs are given in Section 3 subsequently.

\section{Main results}

For $0 \leq u_{0} \in C\left(\mathbb{R}^{n} \backslash B_{1}\right) \cap L^{\infty}\left(\mathbb{R}^{n} \backslash B_{1}\right)$, it follows from the classical theory for parabolic equations that the problem (1)-(3) admits a nonnegative solution locally in time. Moreover, the comparison principle holds for the problem (1)-(3). A solution $u$ to the problem (1)-(3) is said to blow up in a finite time $0<T<+\infty$, if

$$
\|u(\cdot, t)\|_{L^{\infty}\left(\mathbb{R}^{n} \backslash B_{1}\right)}=\sup _{x \in \mathbb{R}^{n} \backslash B_{1}} u(x, t) \rightarrow+\infty \quad \text { as } t \rightarrow T^{-} .
$$

Otherwise, $u$ is said to be global.

The main results of this paper are the following two theorems.

Theorem 2.1 Assume that $1<p<p_{c}=1+\left(2+\lambda_{2}+2 \lambda_{3}\right) /\left(n+\lambda_{1}\right)$. Then each nontrivial nonnegative solution to the problem (1)-(3) blows up in a finite time.

Theorem 2.2 Assume that $p>p_{c}=1+\left(2+\lambda_{2}+2 \lambda_{3}\right) /\left(n+\lambda_{1}\right)$. Then there exist both nontrivial global and blow-up nonnegative solutions to the problem (1)-(3). 


\section{Proof of main results}

First we prove Theorem 2.1 by determining the interactions among the diffusion terms, convection terms and reaction terms by a series of precise integral estimates. Moreover, we need to construct some subsolutions to get some integral estimates of the solutions.

Proof of Theorem 2.1 We prove the theorem by a contradiction argument. Assume that the problem (1)-(3) admits a nontrivial nonnegative global solution $u$. For each $l>1$, set

$$
\psi_{l}(r)= \begin{cases}r^{\lambda_{1}}, & 1 \leq r \leq l, \\ \frac{1}{2} r^{\lambda_{1}}\left(1+\cos \frac{(r-l) \pi}{(\delta-1) l}\right), & l<r<\delta l, \\ 0, & r \geq \delta l,\end{cases}
$$

where $\delta=\pi /\left(n+\lambda_{1}-1\right)+1$. Similar to the proof of Lemma 2.1 in [15], one can show that for each $l>1$,

$$
\begin{aligned}
& \frac{d}{d t} \int_{\mathbb{R}^{n} \backslash B_{1}} u(x, t) \psi_{l}(|x|) d x \\
& \geq--C_{0} l^{-2} \int_{B_{\delta l} \backslash B_{l}} u(x, t) \psi_{l}(|x|) d x \\
& \quad+t^{\lambda_{3}} \int_{\mathbb{R}^{n} \backslash B_{1}}|x|^{\lambda_{2}} u^{p}(x, t) \psi_{l}(|x|) d x, \quad t>0,
\end{aligned}
$$

where $C_{0}=(\delta-1)^{-2} \pi^{2}, B_{l}$ is a ball centered at the origin and with radius $l$ in $\mathbb{R}^{n}$. It follows from the Hölder inequality that

$$
\begin{aligned}
C_{0} & \int_{B_{\delta l} \backslash B_{l}} u(x, t) \psi_{l}(|x|) d x \\
\leq & C_{0}\left(\int_{B_{\delta l} \backslash B_{l}}|x|^{-\lambda_{2} /(p-1)} \psi_{l}(|x|) d x\right)^{(p-1) / p} \\
& \times\left(\int_{B_{\delta l} \backslash B_{l}}|x|^{\lambda_{2}} u^{p}(x, t) \psi_{l}(|x|) d x\right)^{1 / p} \\
\leq & C_{1} l^{n+\lambda_{1}-\left(n+\lambda_{1}+\lambda_{2}\right) / p}\left(\int_{\mathbb{R}^{n} \backslash B_{1}}|x|^{\lambda_{2}} u^{p}(x, t) \psi_{l}(|x|) d x\right)^{1 / p}, \quad t>0,
\end{aligned}
$$

with some constant $C_{1}>0$ depending only on $n, \lambda_{1}, \lambda_{2}$, and $p$. Substitute (6) into (5) to get

$$
\begin{aligned}
\frac{d}{d t} w_{l}(t) \geq & \left(\int_{\mathbb{R}^{n} \backslash B_{1}}|x|^{\lambda_{2}} u^{p}(x, t) \psi_{l}(|x|) d x\right)^{1 / p}\left\{-C_{1} l^{n+\lambda_{1}-2-\left(n+\lambda_{1}+\lambda_{2}\right) / p}\right. \\
& \left.+t^{\lambda_{3}}\left(\int_{\mathbb{R}^{n} \backslash B_{1}}|x|^{\lambda_{2}} u^{p}(x, t) \psi_{l}(|x|) d x\right)^{(p-1) / p}\right\}, \quad t>0,
\end{aligned}
$$

where

$$
w_{l}(t)=\int_{\mathbb{R}^{n} \backslash B_{1}} u(x, t) \psi_{l}(|x|) d x, \quad t \geq 0 .
$$


The Hölder inequality shows

$$
\begin{aligned}
\int_{\mathbb{R}^{n} \backslash B_{1}} u(x, t) \psi_{l}(|x|) d x & \\
\leq & \left(\int_{B_{\delta l} \backslash B_{1}}|x|^{-\lambda_{2} /(p-1)} \psi_{l}(|x|) d x\right)^{(p-1) / p} \\
& \times\left(\int_{\mathbb{R}^{n} \backslash B_{1}}|x|^{\lambda_{2}} u^{p}(x, t) \psi_{l}(|x|) d x\right)^{1 / p} \\
\leq & C_{2} l^{\left(n+\lambda_{1}\right)-\left(n+\lambda_{1}+\lambda_{2}\right) / p}\left(\int_{\mathbb{R}^{n} \backslash B_{1}}|x|^{\lambda_{2}} u^{p}(x, t) \psi_{l}(|x|) d x\right)^{1 / p}, \quad t>0
\end{aligned}
$$

which leads to

$$
\int_{\mathbb{R}^{n} \backslash B_{1}}|x|^{\lambda_{2}} u^{p}(x, t) \psi_{l}(|x|) d x \geq C_{3} l^{\left(n+\lambda_{1}+\lambda_{2}\right)-p\left(n+\lambda_{1}\right)} w_{l}^{p}(t), \quad t>0,
$$

where $C_{2}, C_{3}>0$ are constants depending only on $n, \lambda_{1}, \lambda_{2}$, and $p$. It follows from (8) and (7) that

$$
\begin{aligned}
\frac{d}{d t} w_{l}(t) \geq & \left(C_{3} l^{n+\lambda_{1}+\lambda_{2}-p\left(n+\lambda_{1}\right)}\right)^{1 / p} w_{l}(t)\left\{-C_{1} l^{n+\lambda_{1}-2-\left(n+\lambda_{1}+\lambda_{2}\right) / p}\right. \\
& \left.+C_{3}^{(p-1) / p} l^{(p-1)\left(n+\lambda_{1}+\lambda_{2}\right) / p-(p-1)\left(n+\lambda_{1}\right)} t^{\lambda_{3}} w_{l}^{p-1}(t)\right\}, \quad t>0 .
\end{aligned}
$$

It is noted that the restriction on $p$ in (9) is $p>1$ instead of $1<p<p_{c}$.

Next we prove that there exists a constant $C_{4}>0$ depending only on $u_{0}, n$, and $\lambda_{1}$, such that

$$
w_{l}\left(l^{2}\right)=\int_{\mathbb{R}^{n} \backslash B_{1}} u(x, t) \psi_{l}(|x|) d x \geq C_{4}, \quad l \geq 2 .
$$

Let $v$ be the solution to the following problem:

$$
\begin{aligned}
& \frac{\partial v}{\partial t}=\Delta v+\lambda_{1} \frac{x}{|x|^{2}} \cdot \nabla v, \quad x \in \mathbb{R}^{n} \backslash \bar{B}_{1}, t>0, \\
& \frac{\partial v}{\partial v}=0, \quad x \in \partial B_{1}, t>0, \\
& v(x, 0)=u_{0}(x), \quad x \in \mathbb{R}^{n} \backslash \bar{B}_{1} .
\end{aligned}
$$

Then the comparison principle gives

$$
u(x, t) \geq v(x, t), \quad x \in \mathbb{R}^{n} \backslash B_{1}, t \geq 0 .
$$

Since $0 \leq u_{0} \in C\left(\mathbb{R}^{n} \backslash B_{1}\right) \cap L^{\infty}\left(\mathbb{R}^{n} \backslash B_{1}\right)$ is nontrivial, one can prove by the Green function method that

$$
v(x, 1) \geq C_{5} C_{6}^{-\left(n+\lambda_{1}\right) / 2} \exp \left\{-\frac{(|x|-1)^{2}}{4 C_{6}}\right\}, \quad x \in \mathbb{R}^{n} \backslash \bar{B}_{1},
$$


with some constants $C_{5}, C_{6}>0$ depending only on $u_{0}, n$, and $\lambda_{1}$. Set

$$
\underline{z}(x, t)=C_{5}\left(t-1+C_{6}\right)^{-\left(n+\lambda_{1}\right) / 2} \exp \left\{-\frac{(|x|-1)^{2}}{4\left(t-1+C_{6}\right)}\right\}, \quad x \in \mathbb{R}^{n} \backslash B_{1}, t \geq 1 .
$$

It is easy to verify that $\underline{z}$ is a subsolution to the problem

$$
\begin{aligned}
& \frac{\partial z}{\partial t}=\Delta z+\lambda_{1} \frac{x}{|x|^{2}} \cdot \nabla z, \quad x \in \mathbb{R}^{n} \backslash \bar{B}_{1}, t>1, \\
& \frac{\partial z}{\partial v}=0, \quad x \in \partial B_{1}, t>1, \\
& z(x, 1)=C_{5} C_{6}^{-\left(n+\lambda_{1}\right) / 2} \exp \left\{-\frac{(|x|-1)^{2}}{4 C_{6}}\right\}, \quad x \in \mathbb{R}^{n} \backslash \bar{B}_{1} .
\end{aligned}
$$

The comparison principle, together with (12) and (13), shows that

$$
\begin{aligned}
v(x, t) & \geq \underline{z}(x, t) \\
& =C_{5}\left(t-1+C_{6}\right)^{-\left(n+\lambda_{1}\right) / 2} \exp \left\{-\frac{(|x|-1)^{2}}{4\left(t-1+C_{6}\right)}\right\}, \quad x \in \mathbb{R}^{n} \backslash B_{1}, t \geq 1 .
\end{aligned}
$$

It follows from (11) and (14) that

$$
u(x, t) \geq C_{5}\left(t-1+C_{6}\right)^{-\left(n+\lambda_{1}\right) / 2} \exp \left\{-\frac{(|x|-1)^{2}}{4\left(t-1+C_{6}\right)}\right\}, \quad x \in \mathbb{R}^{n} \backslash B_{1}, t \geq 1,
$$

which implies (10).

Now, taking $t \geq l^{2}$ in (9), one gets

$$
\begin{aligned}
\frac{d}{d t} w_{l}(t) \geq & \left(C_{3} l^{n+\lambda_{1}+\lambda_{2}-p\left(n+\lambda_{1}\right)}\right)^{1 / p} w_{l}(t)\left\{-C_{1} l^{n+\lambda_{1}-2-\left(n+\lambda_{1}+\lambda_{2}\right) / p}\right. \\
& \left.+C_{3}^{(p-1) / p} l^{(p-1)\left(n+\lambda_{1}+\lambda_{2}\right) / p-(p-1)\left(n+\lambda_{1}\right)+2 \lambda_{3}} w_{l}^{p-1}(t)\right\}, \quad t \geq l^{2} .
\end{aligned}
$$

It follows from $p<p_{c}$ that

$$
n+\lambda_{1}-2-\left(n+\lambda_{1}+\lambda_{2}\right) / p<(p-1)\left(n+\lambda_{1}+\lambda_{2}\right) / p-(p-1)\left(n+\lambda_{1}\right)+2 \lambda_{3} .
$$

In addition, (10) yields

$$
\inf \left\{w_{l}\left(l^{2}\right): l \in(2,+\infty)\right\} \geq C_{4} .
$$

Owing to (16) and (17), one sees from (15) that there exist two constants $L>2$ and $\gamma>0$ depending only on $u_{0}, n, \lambda_{1}, \lambda_{2}$, and $\lambda_{3}$ such that, for each $l>L$,

$$
\begin{aligned}
\frac{d}{d t} w_{l}(t) \geq & \left(C_{3} l^{n+\lambda_{1}+\lambda_{2}-p\left(n+\lambda_{1}\right)}\right)^{1 / p} \\
& \times w_{l}(t)\left(\frac{1}{2} C_{3}^{(p-1) / p} l^{(p-1)\left(n+\lambda_{1}+\lambda_{2}\right) / p-(p-1)\left(n+\lambda_{1}\right)+2 \lambda_{3}} w_{l}^{p-1}(t)\right) \\
\geq & \gamma w_{l}^{p}(t), \quad t \geq l^{2} .
\end{aligned}
$$


Thus there exists some $0<T<+\infty$ such that

$$
w_{l}(t)=\int_{\mathbb{R}^{n} \backslash B_{1}} u(x, t) \psi_{l}(|x|) d x \rightarrow+\infty \quad \text { as } t \rightarrow T^{-}
$$

Since supp $\psi_{l}(|x|)=\bar{B}_{\delta l} \backslash B_{1}$, one further gets

$$
\|u(\cdot, t)\|_{L^{\infty}\left(\mathbb{R}^{n} \backslash B_{1}\right)} \rightarrow+\infty \quad \text { as } t \rightarrow T^{-} .
$$

That is to say, $u$ blows up in a finite time.

Remark 3.1 Similar to the proof of critical case in [15] and the proof in Theorem 2.1, one can show that the critical case $p=p_{c}$ is also blow-up case.

We turn to the proof of Theorem 2.2. We use the integral estimate (9) to show the existence of nontrivial blow-up nonnegative solutions, while we construct some global selfsimilar supersolutions to show the existence of nontrivial global nonnegative solutions.

Proof of Theorem 2.2 First consider the blow-up case. As mentioned in the proof of Theorem 2.1, (9) holds for each $p>1$, especially for $p>p_{c}$. In particular, for $l=2$,

$$
\begin{aligned}
\frac{d}{d t} w_{2}(t) \geq & \left(C_{3} 2^{n+\lambda_{1}+\lambda_{2}-p\left(n+\lambda_{1}\right)}\right)^{1 / p} w_{2}(t)\left\{-C_{1} 2^{n+\lambda_{1}-2-\left(n+\lambda_{1}+\lambda_{2}\right) / p}\right. \\
& \left.+C_{3}^{(p-1) / p} 2^{(p-1)\left(n+\lambda_{1}+\lambda_{2}\right) / p-(p-1)\left(n+\lambda_{1}\right)} t^{\lambda_{3}} w_{2}^{p-1}(t)\right\}, \quad t>0,
\end{aligned}
$$

where $w_{2}, C_{1}$, and $C_{3}$ are given in the proof of Theorem 2.1. Let $z$ be the solution to the following problem:

$$
\begin{aligned}
& \frac{\partial z}{\partial t}=\Delta z+\lambda_{1} \frac{x}{|x|^{2}} \cdot \nabla z, \quad x \in \mathbb{R}^{n} \backslash \bar{B}_{1}, t>0, \\
& \frac{\partial z}{\partial v}=0, \quad x \in \partial B_{1}, t>0, \\
& z(x, 0)=C_{7} \exp \left\{-\frac{(|x|-1)^{2}}{4}\right\}, \quad x \in \mathbb{R}^{n} \backslash \bar{B}_{1},
\end{aligned}
$$

where $C_{7}>0$ is a constant to be determined. If

$$
u_{0}(x) \geq C_{7} \exp \left\{-\frac{(|x|-1)^{2}}{4}\right\}, \quad x \in \mathbb{R}^{n} \backslash \bar{B}_{1},
$$

then it follows from the comparison principle that

$$
u(x, t) \geq z(x, t), \quad x \in \mathbb{R}^{n} \backslash B_{1}, t \geq 0 .
$$

Set

$$
\underline{z}(x, t)=C_{7}(t+1)^{-\left(n+\lambda_{1}\right) / 2} \exp \left\{-\frac{(|x|-1)^{2}}{4(t+1)}\right\}, \quad x \in \mathbb{R}^{n} \backslash B_{1}, t \geq 0 .
$$


Then $\underline{z}$ is a subsolution to the problem (19)-(21) and thus the comparison principle gives

$$
z(x, t) \geq \underline{z}(x, t), \quad x \in \mathbb{R}^{n} \backslash B_{1}, t \geq 0 .
$$

It follows from (23)-(25) that

$$
u(x, t) \geq C_{7}(t+1)^{-\left(n+\lambda_{1}\right) / 2} \exp \left\{-\frac{(|x|-1)^{2}}{4(t+1)}\right\}, \quad x \in \mathbb{R}^{n} \backslash B_{1}, t \geq 0 .
$$

Thus, there exists sufficiently large $C_{7}$ such that

$$
C_{3}^{(p-1) / p} 2^{(p-1)\left(n+\lambda_{1}+\lambda_{2}\right) / p-(p-1)\left(n+\lambda_{1}\right)} w_{2}^{p-1}(1) \geq 2 C_{1} 2^{n+\lambda_{1}-2-\left(n+\lambda_{1}+\lambda_{2}\right) / p},
$$

which, together with (18), leads to

$$
\frac{d}{d t} w_{2}(t) \geq \frac{1}{2}\left(C_{3} 2^{n+\lambda_{1}+\lambda_{2}-p\left(n+\lambda_{1}\right)}\right)^{1 / p} C_{3}^{(p-1) / p} 2^{(p-1)\left(n+\lambda_{1}+\lambda_{2}\right) / p-(p-1)\left(n+\lambda_{1}\right)} w_{2}^{p}(t), \quad t \geq 1 .
$$

Then, similar to the discussion in the proof of Theorem 2.1, one sees that if $u_{0}$ satisfies (22), then $u$ blows up in a finite time.

We turn to the global existence case. We prove that the problem (1)-(3) admits a selfsimilar supersolution of the form

$$
\bar{u}(x, t)=(t+1)^{-\alpha} v\left((t+1)^{-\beta}|x|\right), \quad x \in \mathbb{R}^{n} \backslash B_{1}, t \geq 0
$$

if

$$
u_{0}(x) \leq \bar{u}(x, 0), \quad x \in \mathbb{R}^{n} \backslash \bar{B}_{1},
$$

where

$$
\alpha=\frac{2+\lambda_{2}+2 \lambda_{3}}{2(p-1)}, \quad \beta=\frac{1}{2} .
$$

Direct calculations show that if $v$ is a supersolution to the following ordinary differential equation:

$$
v^{\prime \prime}(r)+\frac{n+\lambda_{1}-1}{r} v^{\prime}(r)+\beta r v^{\prime}(r)+\alpha v(r)+r^{\lambda_{2}} v^{p}(r)=0, \quad r>0,
$$

then $\bar{u}$ defined by (26) is a supersolution to equation (1). It follows from $p>p_{c}$ that $0<$ $\frac{2+\lambda_{2}+2 \lambda_{3}}{4(p-1)\left(n+\lambda_{1}\right)}<\frac{1}{4}$, which ensure that we can choose $A>0$ such that

$$
\frac{\alpha}{2\left(n+\lambda_{1}\right)}=\frac{2+\lambda_{2}+2 \lambda_{3}}{4(p-1)\left(n+\lambda_{1}\right)}<A<\frac{1}{4}=\frac{\beta}{2} .
$$

Set

$$
v(r)=\eta \mathrm{e}^{-A r^{2}}, \quad r>0
$$


with $\eta>0$ to be determined. We verify that for sufficiently small $\eta>0, v$ is a supersolution to (28). That is to say,

$$
v^{\prime \prime}(r)+\frac{n+\lambda_{1}-1}{r} v^{\prime}(r)+\beta r^{\lambda_{1}+1} v^{\prime}(r)+\alpha r^{\lambda_{1}} v(r)+r^{\lambda_{2}} v^{p}(r) \leq 0, \quad r>0,
$$

which is equivalent to

$$
4 A^{2} r^{2}-2 A-2\left(n+\lambda_{1}-1\right) A-2 \beta A r^{2}+\alpha+r^{\lambda_{2}} \eta^{p-1} \mathrm{e}^{-A(p-1) r^{2}} \leq 0, \quad r>0
$$

or

$$
2(2 A-\beta) A r^{2}+2\left(\alpha-\left(n+\lambda_{1}\right) A\right)+r^{\lambda_{2}} \eta^{p-1} \mathrm{e}^{-A(p-1) r^{\lambda_{1}+2}} \leq 0, \quad r>0 .
$$

The choice of $A$ shows that (30) holds for sufficiently small $\eta>0$. Therefore, for sufficiently small $\eta>0, \bar{u}$ defined by (26) and (29) is a supersolution to equation (1). Noting

$$
v^{\prime}(r) \leq 0, \quad r>0,
$$

one further sees that $\bar{u}$ is a supersolution to the problem (1)-(3) if $u_{0}$ satisfies (27). Then the comparison principle shows that if $u_{0}$ satisfies (27), the solution to the problem (1)-(3) exists globally.

\section{Competing interests}

The authors declare that they have no competing interests.

\section{Authors' contributions}

All the authors contributed to each part of this study equally and approved the final version of the manuscript.

\section{Author details}

${ }^{1}$ School of Mathematics and Statistics, Beihua University, Jilin 132013, China. ${ }^{2}$ Experimental School of the Affiliated Middle School to the Jilin University, Changchun 130021, China. ${ }^{3}$ School of Mathematics, Jilin University, Changchun 130012, China.

\section{Acknowledgements}

This work is supported by the National Natural Science Foundation of China (11571137) and the Science and technology research project of Jilin Provincial Department of Education (2015-133).

\section{Received: 23 September 2015 Accepted: 15 March 2016 Published online: 23 March 2016}

\section{References}

1. Fujita, $\mathrm{H}$ : On the blowing up of solutions of the Cauchy problem for $\frac{\partial u}{\partial t}=\Delta u+u^{1+\alpha}$. J. Fac. Sci., Univ. Tokyo, Sect. I 13, 109-124 (1966)

2. Andreucci, D, Cirmi, GR, Leonardi, S, Tedeev, AF: Large time behavior of solutions to the Neumann problem for a quasilinear second order degenerate parabolic equation in domains with noncompact boundary. J. Differ. Equ. 174, 253-288 (2001)

3. Andreucci, D, Tedeev, AF: A Fujita type result for a degenerate Neumann problem in domains with noncompact boundary. J. Math. Anal. Appl. 231, 543-567 (1999)

4. Deng, K, Levine, HA: The role of critical exponents in blow-up theorems: the sequel. J. Math. Anal. Appl. 243(1), 85-126 (2000)

5. Levine, HA: The role of critical exponents in blow-up theorems. SIAM Rev. 32(2), 262-288 (1990)

6. Fira, M, Kawohl, B: Large time behavior of solutions to a quasilinear parabolic equation with a nonlinear boundary condition. Adv. Math. Sci. Appl. 11(1), 113-126 (2001)

7. Levine, HA, Zhang, QS: The critical Fujita number for a semilinear heat equation in exterior domains with homogeneous Neumann boundary values. Proc. R. Soc. Edinb., Sect. A 130(3), 591-602 (2000)

8. Qi, YW: The critical exponents of parabolic equations and blow-up in $\mathbb{R}^{n}$. Proc. R. Soc. Edinb., Sect. A 128(1), 123-136 (1998)

9. Qi, YW, Wang, MX: Critical exponents of quasilinear parabolic equations. J. Math. Anal. Appl. 267, 264-280 (2002)

10. Wang, CP, Zheng, SN: Critical Fujita exponents of degenerate and singular parabolic equations. Proc. R. Soc. Edinb., Sect. A 136(2), 415-430 (2006) 
11. Wang, CP, Zheng, SN, Wang, ZJ: Critical Fujita exponents for a class of quasilinear equations with homogeneous Neumann boundary data. Nonlinearity 20(6), 1343-1359 (2007)

12. Winkler, M: A critical exponent in a degenerate parabolic equation. Math. Methods Appl. Sci. 25, $911-925$ (2002)

13. Zhang, QS: A general blow-up result on nonlinear boundary-value problems on exterior domains. Proc. R. Soc. Edinb., Sect. A 131(2), 451-475 (2001)

14. Zheng, SN, Song, XF, Jiang, ZX: Critical Fujita exponents for degenerate parabolic equations coupled via nonlinear boundary flux. J. Math. Anal. Appl. 298, 308-324 (2004)

15. Zheng, SN, Wang, CP: Large time behaviour of solutions to a class of quasilinear parabolic equations with convection terms. Nonlinearity 21(9), 2179-2200 (2008)

16. Azman, I, Jleli, M, Samet, B: Blow-up of solutions to parabolic inequalities in the Heisenberg group. Electron. J. Differ. Equ. 2015, 167 (2015)

17. Jin, C, Yin, J, Zheng, S: Critical Fujita absorption exponent for evolution $p$-Laplacian with inner absorption and boundary flux. Differ. Integral Equ. 27(7-8), 643-658 (2014)

18. Wang, Z, Yin, J: A note on semilinear heat equation in hyperbolic space. J. Differ. Equ. 256(3), 1151-1156 (2014)

19. Wang, C, Zheng, S: Fujita-type theorems for a class of nonlinear diffusion equations. Differ. Integral Equ. 26(5-6), 555-570 (2013)

20. Mazón, JM: Critical Fujita exponent for the diffusion equation in transparent media with source having power-like non-linearities. Adv. Differ. Equ. 18(3-4), 243-264 (2013)

\section{Submit your manuscript to a SpringerOpen ${ }^{\circ}$ journal and benefit from:}

- Convenient online submission

- Rigorous peer review

- Immediate publication on acceptance

- Open access: articles freely available online

- High visibility within the field

- Retaining the copyright to your article 